preasure were detected in all patients with recorded renal lession during the follow up period (in 11/105 pts with lcSSc, and in 8/ 33 pts with dcSSc). Renal failure was significantly more frequent in dcSSc pts ( $p=0,04-\chi 2$ test). In $7(21 \%)$ pts with dcSSc no criteria for pulmonary and renal involvement were present during the follow up period. On the other hand, in 14(13\%) pts with lcSSc pulmonary insufficiency or renal failure were recorded in first 5 years of disease duration.

Conclusion Difusse cutaneous lessions were prognostic sign of lungs and kidneys involvement, as well as pulmonary insufficiency and renal failure in most of the patients, but about $20 \%$ of the dcSSc patients were without severe visceral lessions during the 13 years of follow up period. On the other hand, $13 \%$ of patients with limited cutaneous lessions had bad prognosis and developed pulmonary insufficiency or renal failure in first 5 years of disease duration.

\section{FRI0190 SURGICAL TREATMENT FOR GASTROESOPHAGEAL REFLUX DISEASE IN SYSTEMIC SCLEROSIS}

PD Sampaio-Barros, NA Andreollo, LR Lopes, AG Cunha, AM Samara, NA Brandalise, JF Marques-Neto. Unit of Rheumatology, State University of Campinas, Campinas, Brazil

10.1136/annrheumdis-2001.271

\section{Background}

Objectives To describe the experience with the surgical treatment for gastroesophageal reflux disease (GRD) in systemic sclerosis (SSc).

Methods From 202 patients with the diagnosis of SSc attended at the Scleroderma outpatient clinic in the period 1991-2000, ten patients $(5 \%)$ who presented GRD, with severe chronic esophagitis and stricture, were submitted to surgical treatment. All the patients were female and eight were Caucasian, with seven presenting limited SSc (mean age 53 years and mean disease duration 17 years) and three diffuse SSc (mean age 44 years and mean disease duration 6 years).

Results The correction of the gastroesophageal reflux was performed through videolaparoscopy in nine patients. Seven patients underwent modified Nissen technique and three patients Lind technique. There was satisfactory clinical response, with significant improvement in the heartburn and dysphagia in all the patients. After a mean followup of 34 months (12-76 months), one patient presented clinical relapse (after 54 months) and another patient needed a surgical ressection of a paraesophageal hernia (Y-Roux).

Conclusion The surgical treatment of GRD represents an efficient therapeutic option in SSc patients with severe esophagitis and stricture.

\section{FRI0191 SKIN SCORE IN SYSTEMIC SCLEROSIS}

PD Sampaio-Barros, EM Amstalden, AM Samara, JF Marques-Neto. Unit of Rheumatology, State University of Campinas, Campinas, Brazil

\subsection{6/annrheumdis-2001.272}

\section{Background}

Objectives To report the experience with the use of the Total Skin Score (TSS), in the original (OR-TSS) and modified (MRTSS) Rodnan methods, in systemic sclerosis (SSc), analysing its clinical significance in the model of two (diffuse and limited) and three (diffuse, intermediate and limited) subgroups.
Methods Prospective study analysing skin thickness (assessed using the original and modified Rodnan TSS methods) in 56 patients with SSc. TSS results were compared with SSc clinical variants (divided in the model of two and three subgroups), pathological findings observed in standardised skin biopsies and cutaneous (calcinosis, telangiectasias, pigmentary abnormalities), systemic (articular, vascular, esophageal, pulmonary, cardiac, renal) and laboratory (antinuclear antibody, anticentromere antibody, anti-topoisomerase I antibody) manifestations. Data were analysed on the basis of whether patients had a low TSS $(=<$ 20 ) or a high TSS $(>20)$, in both methods. Logistic regression analysis was performed to determine the independent variables that influenced TSS (in the two methods) and death (after a 5year follow-up).

Results There was statistical association between TSS $=<20$ and limited SSc (both methods, in the SSc model of two subgroups)), grade I skin biopsy (OR-TSS) and calcinosis (MR-TSS). The TSS $>20$ was associated with diffuse SSc (in the SSc models of two and three subgroups), pigmentary abnormalities and articular involvement (both methods) and grade II and III skin biopsy (OR-TSS). There was a statistical trend between TSS $=<$ 20 and calcinosis and anticentromere antibody (OR-TSS), as well as between TSS $>20$ and pulmonary restrictive disease (MRTSS) and anti-topoisomerase I antibody (OR-TSS). The multivariate logistic regression analysis indicated that the clinical variant was the most important factor that influenced significantly TSS (in both methods). In the SSc model of two subgroups, the diffuse variant was associated with TSS $>20$ (both TSS methods) and pigmentary abnormalities, with a statistical trend related to pulmonary restrictive disease and anti-topoisomerase-I antibody, whereas the limited variant was associated with TSS $=$ $<20$, calcinosis and anticentromere antibody. In the SSc model of three subgroups, the diffuse variant was associated with TSS $>20$ (both TSS methods) and pigmentary abnormalities, whereas the limited variant was associated with grade I skin biopsy; the intermediate variant was associated with calcinosis and telangiectasias. After a 5-year follow-up, there were 20 deaths (35.7\%); the multivariate logistic regression analysis indicated that the vascular involvement influenced significantly death, despite the TSS score.

Conclusion TSS, in both original and modified Rodnan methods, represents an useful tool in the management of the skin involvement in SSc, permitting to differentiate distinct patterns in the clinical spectrum of the disease.

\section{FRI0192 LIPID PROFILE IN PATIENTS WITH SYSTEMIC SCLEROSIS}

'J Gozdzik, 'PJ Kotyla, ${ }^{1} E$ J Kucharz, ${ }^{2} \mathrm{~L}$ Brzezinska-Wcislo. ${ }^{1}$ Department of Internal Medicine and Rheumatology; ${ }^{2}$ First Department of Dermatology, Medical University of Silesia, Katowice, Poland

\subsection{6/annrheumdis-2001.273}

Background Systemic sclerosis (SSc) is an inflammatory connective tissue disorder characterised by collagen proliferation, vascular damage and internal organ involvement. It is speculated that vascular changes in SSc patients may be at least partially secondary to impaired lipid metabolism. Hypothyroidism has been reported in patients with SSc and thyroid involvement may additionally effect lipid metabolism.

Objectives The aim of the present study was to evaluate the serum lipid profile in patients with SSc with regard to their thyreometabolic state. 